\title{
SATISFACCIÓN LABORAL, SATISFACCIÓN VITAL Y AUTOESTIMA EN UNA MUESTRA COMPARADA DE SUJETOS CON ESTUDIOS SUPERIORES Y NO SUPERIORES
}

\section{JOB SATISFACTION, LIFE SATISFACTION AND SELF-ESTEEM IN A COMPARED SAMPLE OF SUBJECTS WITH NO HIGHER AND HIGHER EDUCATION}

González Ruiz-Ruano, Verónica (veronicagrr@correo.ugr.es); Fresnedas Blanco, María Josefa (marijosefresnedas@corrreo.ugr.es); Carrillo Pareja, Ana María (amcarrillo@coreo.ugr.es); Martín Rot, Aroa (aroamartin@correo.ugr.es) y García Pereira, Sergio (sergiogp@correo.ugr.es). Universidad de Granada

Artículo dirigido por: Inmaculada Valor Segura. Departamento de Psicología Social. Universidad de Granada

Fecha de recepción: 19 de marzo de 2014.

Fecha de revisión: 19 de marzo de 2014.

Fecha de aceptación: 11 de abril de 2014.

Resumen. En la siguiente investigación nos centramos en investigar cómo la satisfacción laboral, satisfacción vital y autoestima se ven afectadas por el nivel de estudios de una muestra de trabajadores españoles. Siguiendo numerosos estudios como los de Pichler y Wallace (2009), creemos que el nivel educativo superior correlacionará con las tres variables evaluadas. Nuestros datos confirman la hipótesis de que un nivel de estudios superior llevaría a las personas a un nivel de bienestar más elevado, pues encontramos que puntúan más alto tanto en satisfacción laboral como en satisfacción vital, no encontrándose diferencias significativas en cuanto a la autoestima. Palabras clave: satisfacción laboral, satisfacción vital, autoestima.

Abstract. The aim of this research is to look into how affected are job satisfaction, life satisfaction and self-esteem by the studies level of a Spanish workers sample. Following numerous studies like Pichler y Wallace (2009), we think that higher education level will correlate with the three evaluated variables. Our data support the hypothesis that a higher level of studies would lead people to a raised level of well-being, because of we found that they score higher in both job satisfaction and life satisfaction, no significant differences in self-esteem came upon.

Key words: job satisfaction, life satisfaction, self-esteem.

\section{INTRODUCCIÓN}

En una sociedad como en la que actualmente vivimos, el trabajo se transforma en una herramienta de supervivencia y pueden existir diversos factores que afecten a los trabajadores y a sus condiciones de trabajo, lo que puede influir, entre otros, en la satisfacción con la vida, en la satisfacción con el empleo, así como en la autoestima.

Actualmente existe un interés creciente por aspectos relacionados con la calidad de vida de las personas, no sólo en el ámbito general, sino también en el contexto laboral, como puede ser el estudio de la satisfacción laboral (Gamero, 2004). Modelos recientes como el de la Responsabilidad Social Corporativa (RSC), defienden la necesidad de la 
empresa de satisfacer las expectativas de sus empleados, en especial la satisfacción y la calidad de vida (Sánchez, 2008).

La satisfacción laboral puede entenderse como una reacción afectiva hacia el puesto de trabajo, que puede ser el resultado de la comparación, por parte la persona, de los resultados reales que obtiene, con aquellos que son deseados (Cranny et al., 1992). Se trata de una actitud global hacia el trabajo que puede tener causas multidimensionales (Kalleberg, 1977 y González et al., 2011), no estamos ante un concepto absoluto, sino que debe entenderse como un juicio relativo (Gamero, 2004).

Los factores que ayudan a establecer los niveles de satisfacción en el trabajo son numerosos; desde el tiempo de vacaciones anuales, hasta el reconocimiento por parte del superior, acceso a un ascenso, etc. Munchinsky (1983) afirmó que la satisfacción laboral está formada por dos tipos de factores. Por un lado, la satisfacción intrínseca, donde se recogen las experiencias de los individuos con su trabajo y las recompensas con valor interno (por ejemplo, factores de reconocimiento, responsabilidad, promoción). En el otro extremo, se sitúan los factores relacionados con la satisfacción extrínseca (el salario, las políticas de la compañía, los recursos, la supervisión, etc.) (en González et al., 2011).

De este modo se pasa de estudiar únicamente aspectos extrínsecos al individuo, para centrar la atención también en aspectos intrínsecos, en los que participan percepciones individuales, como por ejemplo, satisfacción vital y autoestima.

La autoestima es un concepto ampliamente estudiado en el contexto laboral, Ganster y Schaubroeck (1995) definen la autoestima laboral como "una actitud individual sobre la competencia, desempeño y valor profesional a lo largo de una dimensión positivanegativa, constituye un factor importante en la explicación del desempeño, la satisfacción laboral y vital."

Se ha demostrado que el nivel de autoestima es un fuerte predictor de nuestros logros o fracasos; puntuaciones altas favorecerán al desarrollo de habilidades y al nivel de seguridad personal (Mora, 2010). Estos factores, a su vez, se verán reflejados en la calidad del trabajo de los empleados; a mayor autoestima, mayor rendimiento y satisfacción, tanto con la vida, como con su puesto de trabajo. Una baja autoestima no solo repercutirá en la cantidad y la calidad del trabajo, sino que afectará a las relaciones sociales y laborales (jefe, clientes, proveedores, compañeros...).

Así mismo, se ha observado que el tipo de ocupación y el puesto de trabajo, pueden ser aspectos determinantes en la satisfacción que posteriormente informará un empleado. Siendo así, se han conseguido explicar muchas de las diferencias en satisfacción laboral, apelando a condiciones laborales objetivas, como por ejemplo, el tipo de ocupación (Pichler y Wallace, 2009). Algunas investigaciones parecen indicar que cuanto mayor sea la posición jerárquica, mayor nivel de satisfacción general se encontrará (López et al., 2010; Pichler y Wallace, 2009). Esta satisfacción se manifestará, a su vez, en variables de carácter intrínseco, como la participación en las decisiones, la autoestima y la satisfacción específica con la tarea. 
Por otro lado, Campbell et al. (1976) demostraron que entre un $15 \%$ y un $50 \%$ de la varianza obtenida de la satisfacción con la vida podía explicarse mediante la satisfacción laboral. Wright (2006), reafirmó esta idea, señalando que posiblemente la satisfacción laboral sea el indicador más importante de la satisfacción con la vida.

Debemos tener en cuenta que nuestro bienestar emocional cumple funciones de carácter autorregulador, tanto a nivel emocional como conductual (Hervás y Vázquez, 2006). De este modo, se ha observado que la satisfacción con la vida correlaciona fuertemente con logros personales y con aspectos sociales, como el nivel educativo (Diener et al., 2009; Kahneman et al., 2006; Kahneman y Stone, 2010).

Actualmente, la situación de crisis, está haciendo que el trabajo se considere no sólo una necesidad, sino una condición cooperante con el estado de bienestar. Los estudios de evolución vital han puesto de manifiesto que la vida laboral constituye uno de los tres roles más característicos de la etapa adulta, junto con la vida en pareja y la maternidad (Atchley, 1975; Bromley, 1966; Havighurst, 1982; Havighurst, 1952; Levinson, 1977 y Neugarten y Hagestad, 1976). El rol laboral se convierte tanto en una exigencia, como en un síntoma de ajuste al sistema social, lo que le acabará favoreciendo un buen funcionamiento de los mecanismos de ajuste psicológico (Jahoda, 1981, 1987).

\section{BASE EPISTEMOLÓGICA}

\subsection{Objetivo e hipótesis}

Los objetivos de este estudio son dos. El primero es comprobar que el nivel de estudios, diferenciando entre superiores y no superiores, afecta a la satisfacción laboral, a la satisfacción vital y a la autoestima. El segundo objetivo, es averiguar si estas tres variables correlacionan entre sí de manera significativa.

De acuerdo con la proposición conjetural de Kerlinger y Lee (2001), las personas con un nivel de estudios superior presentará mayor satisfacción laboral, mayor satisfacción vital y mayor autoestima que las personas con un nivel de estudios no superior.

\subsection{Método}

\section{- Muestra}

En nuestro estudio hemos utilizado una muestra compuesta por 90 sujetos (43 hombres y 47 mujeres) con una media de edad de 37,37 (DT=10,55) separados en dos grupos, estudios superiores (43 sujetos) y estudios no superiores (47 sujetos). Para seleccionar a los sujetos se ha utilizado un muestreo casual o intencional, cuyos límites son: estar en edad laboral, ser mayor de edad (18-67 años) y estar trabajando actualmente.

\section{- Material}

Para evaluar las diferentes variables se elaboró un cuadernillo compuesto de los siguientes cuestionarios: 
Cuestionario de satisfacción laboral S20/23 (Meliá y Peiró, 1998): Este cuestionario, con una consistencia interna de .96, está compuesto por 23 ítems que se responden en una escala de 1 a 7 desde totalmente insatisfecho hasta totalmente satisfecho. El cuestionario incluye 5 dimensiones.

Escala de autoestima de Rosenberg (RSES; Rosenberg, 1989): La escala está compuesta por 10 ítems, 5 formulados en positivo y 5 en negativo para controlar la aquiescencia. Estos ítems se contestan en una escala de 4 que va desde muy de acuerdo a muy en desacuerdo. La consistencia interna de esta escala se encuentra entre .76 y .87 y su fiabilidad es de .8 .

Escala de satisfacción vital (SWLS; Diener et al., 1985): Escala compuesta por cinco ítems que deben ser contestados en una escala tipo likert de 7 puntos que va desde fuertemente en desacuerdo a fuertemente de acuerdo. El coeficiente a de Cronbach oscila entre .85 y .89 para los diferentes ítems.

Datos demográficos que incluyen edad, sexo, trabajo, nivel de formación y formación mínima que se le exige en su trabajo.

\section{Procedimiento}

Los cuadernillos fueron pasados a los sujetos de forma presencial en sus domicilios, habiendo sido avisados antes sobre qué iban a contestar. Se recogieron los datos de satisfacción vital, satisfacción laboral, autoestima y datos demográficos.

Hemos llevado a cabo un análisis estadístico realizando una comparación de muestras independientes de los grupos de estudios superiores e inferiores y una correlación de Pearson bivariada entre las 3 variables dependientes (satisfacción laboral, satisfacción vital y autoestima).

\section{RESULTADOS}

En un análisis de medias y desviación típica, se ha encontrado que los sujetos con estudios superiores puntúan más alto en las escalas de Satisfacción Laboral, con una media de 5,07 (DT=1,11), Satisfacción con la Vida con una media de 23,95 (DT=5,85) y Autoestima con una media de $33,69(\mathrm{DT}=4,39)$ versus a los sujetos con estudios no superiores, cuyas medias son 4,46 (DT=1,14), 21,14 (DT=6,22) y 32,61 (DT=4,04), respectivamente. Estos datos se observan en la Tabla 1.

\begin{tabular}{|c|c|c|}
\hline \multicolumn{3}{|c|}{$\begin{array}{l}\text { Tabla } 1 \\
\text { Puntuaciones medias en cada una de las escalas en función del nivel de estudios de los } \\
\text { sujetos }\end{array}$} \\
\hline & Superiores & No Superiores \\
\hline Satisfacción Laboral & 5,079 & 4,46 \\
\hline Satisfacción con la Vida & 23,95 & 21,14 \\
\hline Autoestima & 33,69 & 32,31 \\
\hline
\end{tabular}


Gráfica 1.

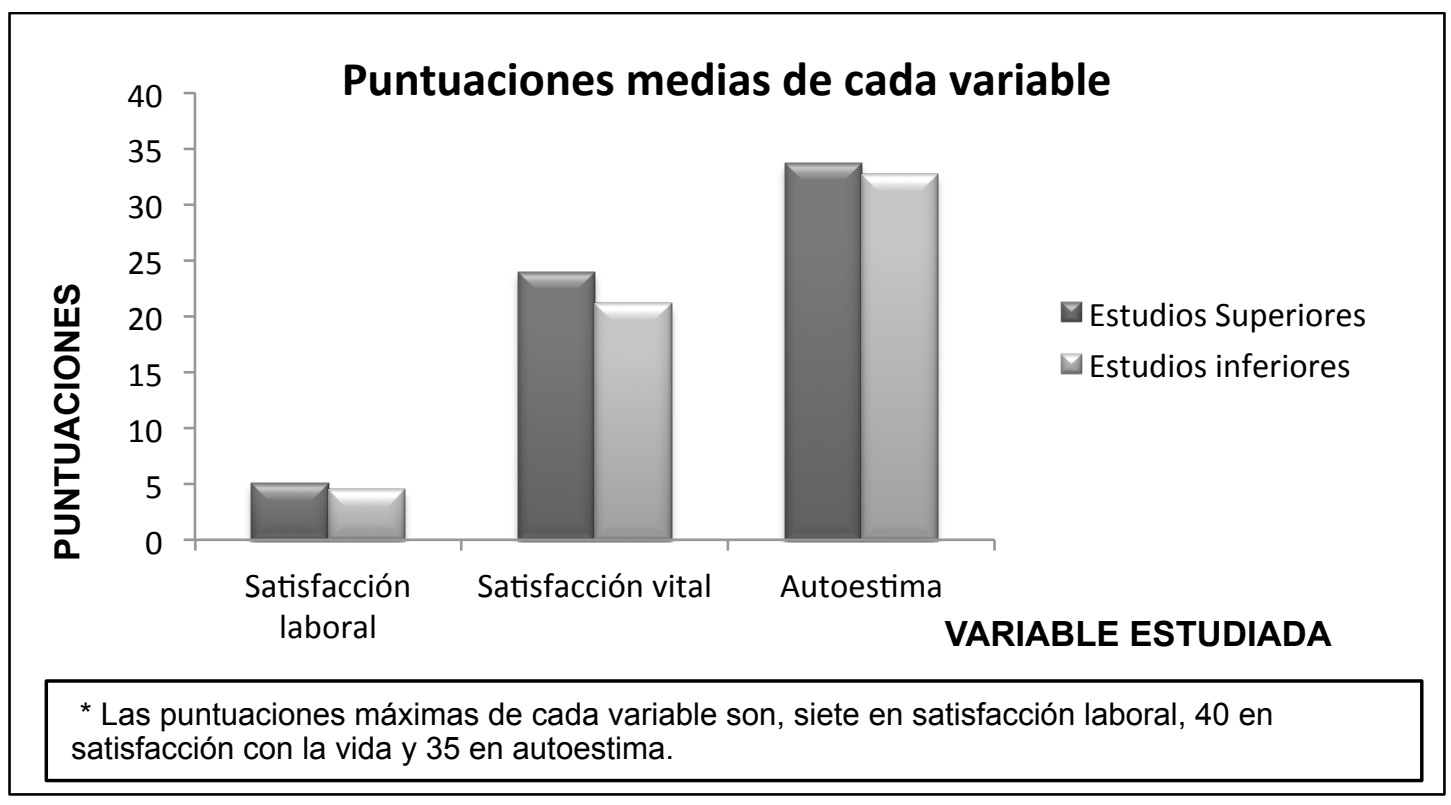

Para observar si hay diferencias significativas entre ambos grupos de sujetos, en relación a estas tres dimensiones, se realizó una prueba $t$ student para muestras independientes, encontrándose diferencias significativas en la satisfacción laboral informada por los sujetos entre los sujetos con estudios superiores y los sujetos con estudios no superiores $(\mathrm{t}(88)=2,58 ; \mathrm{p}<0,05)$, y en satisfacción vital también se han encontrado diferencias significativas $(t(88)=2,19 ; p<0,05)$. Por último, no se han encontrado diferencias significativas en autoestima entre ambos grupos, aunque la media de los sujetos con estudios superiores es mayor $(t(88)=1,21 ; p>0,05)$. Véase Tabla 2.

\begin{tabular}{ccccccc}
\hline \multicolumn{7}{c}{ Tabla 2 } \\
Comparación de medias con una prueba t de student para muestras \\
independientes
\end{tabular}

Para finalizar, se ha llevado a cabo un análisis correlacional de Pearson para observar cómo las tres variables correlacionan entre sí. Se ha encontrado que la satisfacción laboral correlaciona con la satisfacción vital $(r(90)=0,46 ; p<0,01)$ y con la autoestima $(r(90)=0,49 ; p<0,01)$ y la satisfacción vital también correlaciona con la autoestima $(r(90)=0,61 ; p<0,01)$. Las tres variables correlacionan positivamente, encontrándose que cuando aumente una, aumentarán las otras dos, y viceversa. Véase Tabla 3. 


\begin{tabular}{llrrr}
\hline & Correlación de Pearson para las tres variables dependientes & \\
& & $\begin{array}{c}\text { Satisfacción } \\
\text { Laboral }\end{array}$ & $\begin{array}{c}\text { Satisfacción } \\
\text { vital }\end{array}$ & Autoestima \\
\hline Satisfacción & Correlación de & 1 &, $458\left(^{* *}\right)$ &, $487\left(^{* *}\right)$ \\
Laboral & Pearson & &, 000 &, 000 \\
& Sig. (bilateral) & 90 & 90 & 90 \\
Satisfacción & Correlación de &, $458\left(^{* *}\right)$ & 1 &, $610\left(^{* *}\right)$ \\
Vital & Pearson &, 000 & &, 000 \\
& Sig. (bilateral) & 90 & 90 & 90 \\
Autoestima & N &, $487\left(^{* *}\right)$ &, $610\left({ }^{* *}\right)$ & 1 \\
& Correlación de &, 000 &, 000 & \\
& Pearson & &
\end{tabular}

\section{DISCUSIÓN}

Los resultados obtenidos parecen estar en consonancia con nuestra hipótesis, de esta manera, los sujetos con estudios superiores presentan una mayor satisfacción laboral y vital que los sujetos con estudios no superiores. En el caso de la autoestima, pese a que no existen diferencias significativas, se puede observar que la media de puntuaciones en esta variable es mayor en aquellos sujetos con estudios superiores.

Según Diener et al. (2009), el nivel educativo puede funcionar como un buen predictor de la satisfacción vital, mostrando que a medida que se ocupan puestos más altos en la jerarquía de la organización, mayor va a ser el bienestar personal de la persona. Nuestros resultados son consistentes con esta teoría, mostrando que aquellos sujetos con estudios superiores, obtienen mayor puntuación en la escala de Satisfacción Vital. Así mismo, se ha observado que atendiendo al nivel educativo, las puntuaciones en nuestras variables siempre son más altas en personas con niveles educativos superiores. (En la Gráfica 1. se pueden observar las diferencias entre las medias de las tres variables).

Una posible explicación a nuestros resultados puede encontrarse en la interacción de las tres variables, explicación que, a su vez, sería consistente con los resultados hallados a partir de nuestra muestra de sujetos. Existe una correlación significativa y positiva entre satisfacción laboral, satisfacción con la vida y autoestima, es decir, que a medida que una aumenta, el resto aumentan también. Campbell et al. (1976), afirmó que la satisfacción laboral podía ser un fuerte indicador de la satisfacción vital, de modo que aquellas personas que se sienten bien en su trabajo y están orgullosas de lo que hacen, tenderán a mostrar actitudes más positivas hacia su vida.

Así mismo, las puntuaciones altas en autoestima favorecerán que los sujetos se muestren más satisfechos en general, tanto con su situación laboral como con su vida. Como ya predijo Mora (2010), cuanto mayor sea la autoestima, más capaces y seguras se sentirán las personas con respecto a todo lo que realicen, lo que les permitirá sentirse personas íntegras y competentes. 
Otra posible explicación puede consistir en que las personas con niveles educativos superiores tienden a desempeñar puestos de trabajo en los que las situaciones laborales, el clima de la empresa, el salario, las oportunidades de promoción, las vacaciones, etc., son mejores y más deseables, mientras que aquellas personas que no han recibido dicha formación, suelen tener condiciones más precarias. Pichler y Wallace (2009) ya informaron de que muchas de las diferencias en la satisfacción laboral, y por ende, en la satisfacción vital, pueden ser explicadas por las condiciones de trabajo a las que las personas están expuestas.

En una situación como la actual, datos como los que aquí se recogen deberían ser tenidos en cuenta en todas las empresas del mercado, ya que tienen en sus manos mejorar, no sólo su actividad económica a través de la mejora del rendimiento de sus trabajadores, sino que pueden mejorar la calidad de vida de millones de personas que se encuentran ahora mismo en situación de empleo y favoreciendo que cada vez vivamos en un mundo con más y mejores condiciones para las personas.

En conclusión, los datos favorecen a las teorías que se proponen en la literatura especializada, las personas con niveles educativos altos van a mostrar mejores puntuaciones en las tres variables estudiadas (Diener et al., 2009; Kahneman et al., 2006; Kahneman y Stone, 2010). A su vez, todas y cada una de estas variables van a ejercer influencias recíprocas entre ellas, de manera que cuanto más aumente una, más lo harán las demás.

Por último, debemos dejar claro que este estudio tiene también sus limitaciones, pues existen diversos aspectos que han de tenerse en cuenta. Al no ser una muestra representativa, no pueden sacarse conclusiones generalizables a toda la población española. Además, el contexto en el que se les ha pasado puede estar funcionando como variable extraña no controlada, puesto que no ha sido administrado en una situación de laboratorio donde estas variables puedan quedar igualadas. Así mismo, tampoco se han controlado aquellas situaciones en las que los sujetos tenían un nivel de estudios superior, pero estaban desempeñando un puesto de trabajo que no necesitaba de dicha formación, lo que puede distorsionar los datos obtenidos. De esta forma, pueden ser necesarias nuevas líneas de investigación que sigan el hilo de nuestro trabajo, que además de controlar estas variables, traten de introducir otras nuevas, como por ejemplo diferencias de sexo, edad, motivación, burnout y, lo que consideramos más importante, que traten de diferenciar, no sólo entre estudios superiores y no superiores, sino entre los puesto de la jerarquía que cada persona ocupa.

\section{REFERENCIAS}

Asenjo Fenoy, A. De Dios Chacón, M., y Banqueri López, M., (2012). Cualificación y satisfacción laboral: un estudio sobre los empleos para los que hemos sido formados previamente. ReiDoCrea, 1, 12-20.

Atchley, R. (1975). The life course, age grading, and age-linked demands for decision making. En D. Natan y L. Ginsberg (Eds.), Life-span developmental psychology. Normative life crises. Nueva York: Academic Press. pp.261-278. 
Atienza, F.L., Pons, D., Balaguer, I., y García-Merita, M., (2000). Propiedades Psicométricas de la Escala de Satisfacción con la Vida en Adolescentres. Psicothema. 12 (2), 314-319.

Blanch, J.M., Sahagún, M., Cantera, L., y Cervantes, G., (2010). Cuestionario de Bienestar Laboral General: Estructura y Propiedades Psicométricas. Revista de Psicología del Trabajo y de las Organizaciones, 26(2), 157-170.

Bória, S., Crespi, M., y Mascarilla, O., (2012). Variables determinantes de la satisfacción laboral en España. Cuadernos de economía, 35, 9-16.

Bromley, D. (1966). The Psychology of Human Aging. Baltimore: Penguin.

Campbell, A., Converse, P.E., y Rodgers, W.L. (1976). The quality of American life: perceptions, evaluations and satisfaction. Russell Sage Foundation, New York: Wiley.

Chiang, M.M., Salazar, C.M., y Núñez, A. (2007). Clima organizacional y satisfacción laboral en un establecimiento de salud estatal: hospital tipo 1. Theoria, 16 (2), 61-76.

Cramer, D. (1995). Life and Job Satisfaction: A Two-Wave Panel Study. The Journal of Psychology, 129 (3), 261-267.

Cranny, C.J., Smith, P.C., y Stone, E.F. (1992). Job satisfaction: how people feel about their jobs and how it affects their performance. Nueva York: Lexington Books, An Imprint of Macmillan.

Diener, E., Emmons, R., Larsen, R.J., y Griffin, S. (1985). The Satisfaction With Life Scale. Journal of Personality Assessment, 49, 71-75.

Diener, E., Kahneman, D., Arora, R., Harter, J., y Tov, W. (2009). Income's differential influence on judgments of life versus affective well-being. En E. Diener (Ed.), Assessing well-being: The collected works of Ed Diener (pp. 233-245). Dordrecht, Netherlands: Springer.

Estévez-Guerra, G.J., Núñez-González, E., Fariña-López, E., Marrero-Medina, C.D., y Hernández-Marrero, P. (2007). Determinantes de la satisfacción laboral en centros geriátricos. Rev Esp Geriatr Gerontol, 42(5), 285-292.

Gamero Burón, C. (2004). Satisfacción laboral de los asalariados en España. Especial referencia a las diferencias por género. Cuadernos de Economía, 27, 109-146.

Ganster, D.C. y Schaubroeck, J. (1995). The moderating effect of self-steem an the work stress-employee health relationship. En R. Crandall y P. Perrewe (Eds.), Occupational Stress: A Handbook (2a ed.) (pp. 167-177). Nueva York: Taylor \& Francis.

González Santa Cruz, F., Sánchez Cañizares, S.M., y López-Guzmán, T. (2011). Satisfacción laboral como factor crítico para la calidad: el caso del sector hostelero de la provincia de Córdoba-España. Estudios y Perspectivas en Turismo, 20, 1047-1068. 
Hart, P., (1999). Predicting Employee Life Satisfaction: A Coherent Model of Personality, Work and Nonwork Experiences, and Domain Satisfactions. Journal of applied psychology, 84 (4), 564-584.

Havighurst, R.J. (1982). The world of work. En B. Wolman (Ed.), Handbook of Developmental Psychology. Englewood Cliffs, N.J.: Prentice-Hall.

Havighurst, R. (1952). Developmental tasks and education. Nueva York: David McKay.

Hervás, G., y Vázquez, C. (2006). La regulación afectiva: Modelos, investigación e implicaciones para la salud mental y física. Revista de Psicología General y Aplicada, 59, 9-32.

Jahoda, M. (1981). Work, employment and unemployment: Values, theories, and approaches in social research. American Psychologist, 36, 184-191.

Jahoda, M. (1987). Empleo y desempleo: un análisis psico-sociológico. Madrid: Morata.

Jiménez, A., Jara, M.J., y Miranda, E. (2012). Burnout, apoyo social y satisfacción laboral en docentes. Revista semestral de la Asociación Brasileña de Psicología Escolar y Educacional, 16(1), 125-134.

Kalleberg, A.L. (1977). Work values and job rewards: A theory of job satisfaction. American Sociological Review 42 (1), 124-143.

Kahneman, D., Krueger, A., Schkade, D., Schwarz, N., y Stone, A. (2006). Would you be happier if you were richer? A focusing illusion. Science, 312, 1908-1910.

Kahneman, D., y Stone, A. (2010). High income improves evaluation of life but not emotional well-being. National Academy of Sciences of the United States of America (PNAS), 107, 16489-16493.

Kerlinger, F.N., y Lee, B. (2001). Howard: Investigación del comportamiento. Métodos de Investigación en Ciencias Sociales. México: Mc Graw Hill, $4^{\mathrm{a}}$ ed.

Levinson, D. (1977). Middle adulthood in modern society: A sociopsychological view. En D. Di Renzo (Ed.), We the people: Social change and social character. Wesport, Conn.: Greenwood Press.

López-Guzmán, T.J., Sánchez Cañizares, S.M., y Nascimento Jesús, M.M. (2010). La satisfacción laboral como valor intangible de los recursos humanos. Un estudio de caso en establecimientos hoteleros. Teoría y Praxis, 7, 35-53.

McKay, M., y Fanning, P. (1987). Autoestima: evaluación y mejora. Barcelona: Ediciones Mártinez Roca, S.A.

Meliá, J.L., Peiró, J.M., (1989). La medida de la satisfacción laboral en contextos organizacionales: El Cuestionario de Satisfacción S20/23. Psicologemas, 5, 5974.

Mora, C. (2010). Autoestima y asertividad en el trabajador y gerencia venezolana. Recuperado de http://entorno-empresarial.com/articulo/4850/autoestima-yasertividad-en-el-trabajador-y-gerencia-venezolana. 
Neugarten, B., y Hagestad, G. (1976). Age and life course. En R. Binstork y E. Shanes (Eds.), Handbook of aging and social sciences. Nueva York: Van Nostrand.

Pichler, F., Wallace, C. (2009). What are the Reasons for differences in job satisfaction across Europe? Individual, compositional, and institutional explanations. European Sociological Review, 25(5), 535-549.

Polo, J. D., Fernández, M., y Ramírez, R. G. (2012). Diseño del trabajo y la satisfacción con la vida. Revista Venezolana de Gerencia, 17(59), 466-481.

Sánchez, C. (2008). Motivación, satisfacción y vinculación. ¿Es gestionable la voluntad de las personas en el trabajo? Acción Psicológica, 5 (1), 9-28.

Vázquez, C., Duque, A., y Hervás, G. (2012). Escala de Satisfacción con la Vida (SWLS) en una muestra representativa de españoles adultos: Validación y datos normativos. Madrid.

Vázquez, A.J., Jiménez, R., y Vázquez-Morejón, R. (2004). Escala de autoestima de Rosenberg: fiabilidad y validez en población clínica española. Apuntes de Psicología. 22 (2), 247-255.

Wright, T. (2006). The emergence of job satisfaction in organizational behavior: A historical overview of the dawn of job attitude research. Journal of Management History, 12, 262-271. 